\title{
Interlayer Trions in the MoS2/WS2 van der Waals Heterostructure
}

\author{
Deilmann, Thorsten; Thygesen, Kristian Sommer
}

Published in:

Nano Letters

Link to article, DOI:

10.1021/acs.nanolett.7b05224

Publication date:

2018

Document Version

Peer reviewed version

Link back to DTU Orbit

Citation $(A P A)$ :

Deilmann, T., \& Thygesen, K. S. (2018). Interlayer Trions in the MoS $/ \mathrm{WS}_{\text {, van }}$ der Waals Heterostructure. Nano Letters, 18(2), 1460-1465. https://doi.org/10.1021/acs.nanolett.9b05z24

\section{General rights}

Copyright and moral rights for the publications made accessible in the public portal are retained by the authors and/or other copyright owners and it is a condition of accessing publications that users recognise and abide by the legal requirements associated with these rights.

- Users may download and print one copy of any publication from the public portal for the purpose of private study or research.

- You may not further distribute the material or use it for any profit-making activity or commercial gain

- You may freely distribute the URL identifying the publication in the public portal

If you believe that this document breaches copyright please contact us providing details, and we will remove access to the work immediately and investigate your claim. 


\title{
Interlayer trions in the $\mathbf{M o S}_{2} / \mathbf{W S}_{2}$ van der Waals heterostructure
}

\author{
Thorsten Deilmann ${ }^{* \dagger}$ and Kristian Sommer Thygesen ${ }^{\dagger}$ \\ $\dagger C A M D$, Department of Physics, Technical University of Denmark, DK-2800 Kongens \\ Lyngby, Denmark \\ $\ddagger$ Center for Nanostructured Graphene (CNG), Technical University of Denmark, DK-2800 \\ Kongens Lyngby, Denmark \\ E-mail: thorsten.deilmann@wwu.de \\ Phone: +45 45253188
}




\begin{abstract}
Electronic excitations in van der Waals heterostructures can have interlayer or intralayer character depending on the spatial localisation of the involved charges (electrons and holes). In the case of neutral electron-hole pairs (excitons), both types of excitations have been explored theoretically and experimentally. In contrast, studies of charged trions have so far been limited to the intralayer type. Here we investigate the complete set of interlayer excitations in a $\mathrm{MoS}_{2} / \mathrm{WS}_{2}$ heterostructure using a novel ab-initio method, which allows for a consistent treatment of both excitons and trions at the same theoretical footing. Our calculations predict the existence of bound interlayer trions below the neutral interlayer excitons. We obtain binding energies of $18 / 28 \mathrm{meV}$ for the positive/negative interlayer trions with both electrons/holes located on the same layer. In contrast, a negligible binding energy is found for trions which have the two equally charged particles on different layers. Our results advance the understanding of electronic excitations in doped van der Waals heterostructures and their effect on the optical properties.
\end{abstract}

\title{
Keywords
}

2D materials, heterostructures, interlayer excitons, interlayer trions, optional spectra, manybody perturbation theory 
Monolayers of group VI transition metal dichalcogenides (TMDCs), are direct band gap semiconductors with a plethora of unique optical properties. ${ }^{116}$ In monolayers excitons are naturally confined to a single layer. In multilayer or bulk systems, new types of excitons can form with electron and holes distributed over different layers. Such interlayer excitons are potential candidates for realizing a wealth of interesting physical phenomena, such as Bose-Einstein condensation, high temperature superfluidity, dissipationless current flow, and the light-induced exciton spin Hall effect. ${ }^{7} \cdot 9$

Interlayer excitons can form in homogeneous layered structures (e.g. bulk $\mathrm{MoS}_{2}, \mathrm{MoSe}_{2}$, or $\left.\mathrm{MoTe}_{2} \underline{10111}\right)$ or artificial heterostructures which are build up by stacking monolayers of different two-dimensional materials. $\frac{12}{15}$ Depending on the exact band alignment in the heterostructure, the additional interlayer states may become the lowest optically active exciton. $\frac{15}{15}$

In addition to neutral excitons, the formation of charged trions can influence or even dominate the photoluminescence spectra when free charges are present. $\frac{[16[20}{2}$ Such additional charges can stem from gating, defects or a substrate. Thus the trions can be charged positively or negatively. Recently Mouri et al. ${ }^{21}$ have ascribed certain features in the photoluminescence spectrum of $\mathrm{MoS}_{2} / \mathrm{MoSe}_{2}$ to trion states with interlayer character. On the other hand Miller et al. ${ }^{22}$ interpreted the same features to arise from the coupling of the neutral interlayer exciton with phonons.

In this study we focus on the intra- and interlayer character of zero-momentum (direct) excitons and trions in an prototypical $\mathrm{MoS}_{2}-\mathrm{WS}_{2}$ heterostructure because finite momentum (indirect) excitations do not have optical amplitudes at low temperatures. Before we discuss the possible trion states we analyse the electronic structure as well as the neutral excitons. Using first-principles GW+BSE calculations ${ }^{23}$ we unambiguously identify an optically active interlayer exciton when both layers are rotationally aligned and lattice matched. Having discussed this, we use our ab-initio approach ${ }^{24}$ to describe and investigate the properties of positive and negative (intra- and) interlayer trions. To distinguish between intra- and 


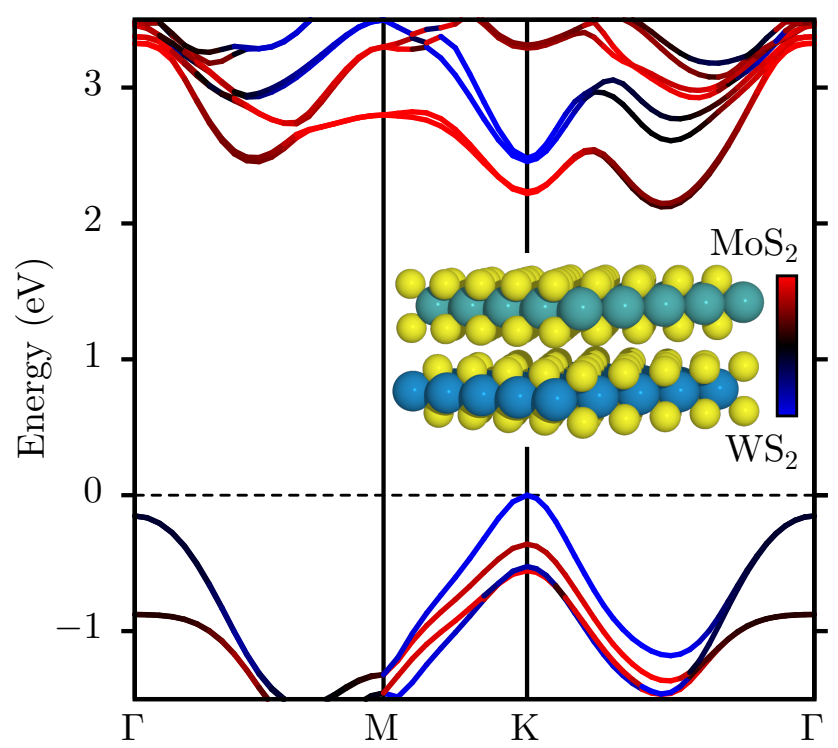

Figure 1: Band structure of a $\mathrm{MoS}_{2}-\mathrm{WS}_{2}$ heterostructure (structure shown in the insert) from the $G d W(\mathrm{LDA})$ approximation. ${ }^{30}$ The color of the band reveals the character of the monolayer, i.e. red for the upper $\mathrm{MoS}_{2}$ layer, blue for $\mathrm{WS}_{2}$, and black for strong hybridisation.

interlayer states experimentally, it is possible to apply a perpendicular electric field. For small fields, we find the same linear dependency of interlayer excitons and trions on the field strength. To our knowledge, this is the first study of interlayer trions.

Several different heterostructures have so far been fabricated, including heterobilayers of $\mathrm{MoSe}_{2}-\mathrm{WSe}_{2}{ }^{15 / 25 \mid 26}$ and $\mathrm{MoS}_{2}-\mathrm{WS}_{2} \cdot{ }^{13 \mid 27}$ In the optical spectrum of these systems additional peaks below the intralayer excitons have been observed, which have been assigned to interlayer excitons. Here we focus on the $\mathrm{MoS}_{2}-\mathrm{WS}_{2}$ heterobilayer, because of the very good lattice match (the lattice constant of $\mathrm{WS}_{2}$ is $a_{\text {lat }}^{\mathrm{WS}_{2}}=3.155 \AA^{28}$ and thus only $0.2 \%$ smaller compared to $\mathrm{MoS}_{2} a_{\text {lat }}^{\mathrm{WS}_{2}}=3.160 \AA^{29}$ ) making it suitable for ab-initio calculations. In this study we neglect this difference and we also use the $\mathrm{MoS}_{2}$ lattice constant of $a=3.16 \AA$ for $\mathrm{WS}_{2}{ }^{1}$

The band structure of the $\mathrm{MoS}_{2}-\mathrm{WS}_{2}$ heterostructure is shown in Fig. 1. The contribution to the electronic states from the $\mathrm{MoS}_{2}$ and $\mathrm{WS}_{2}$ layers is indicated by red and blue, respectively. As previously observed ${ }^{13 \mid 27}$ we find the heterobilayer has Type II band alignment with

\footnotetext{
${ }^{1}$ We note that the usage of the $\mathrm{WS}_{2}$ lattice constant $(0.2 \%$ smaller $)$ only leads to small quantitative differences which are not further discussed here.
} 
the conduction band (CB) of $\mathrm{MoS}_{2}$ about $0.24 \mathrm{eV}$ below that of $\mathrm{WS}_{2}$ and the valence band (VB) of of $\mathrm{WS}_{2} 0.36 \mathrm{eV}$ above that of $\mathrm{MoS}_{2}$. We note that this alignment is crucial for the existence of low lying interlayer excitations which mostly build up by contributions around $\mathrm{K}$ (for discussion see below). At K our $G d W$ calculations yield a gap of $2.28 \mathrm{eV}$ (between bands of different layers), while for the intralayer band gaps we obtain $2.74 \mathrm{eV}$ for $\mathrm{MoS}_{2}$ and $2.61 \mathrm{eV}$ for $\mathrm{WS}_{2}$, respectively. Note that these values are slightly red shifted compared to the free standing monolayers because of the additional screening in the bilayer structure. $\underline{3132}$

Although the bands mostly keep the character of the original single layer around $\mathrm{K}$, a small hybridisation between the upper $\mathrm{MoS}_{2} \mathrm{VB}$ and the lower $\mathrm{WS}_{2} \mathrm{VB}$ is observed. We note that the heterobilayer is an indirect semiconductor (like bilayers of $\mathrm{MoS}_{2}$ and $\mathrm{WS}_{2}$ ) with a $\mathrm{CB}$ minimum between $\mathrm{K}$ and $\Gamma$. The character of the corresponding wave function at the CB minimum shows a strong hybridisation between the layers, but this is not important for the present study which focuses on the $q=0$ excitations of which the lowest are formed by vertical transition at $\mathrm{K}$.

To calculate the excitons of the heterostructure from first principles we employ many-

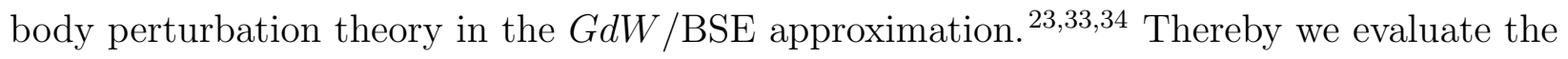
optical spectrum of the heterostructure, see Fig. 2(a). The excitations related to the single monolayers $\mathrm{A}_{\mathrm{WS}_{2}}$ and $\mathrm{A}_{\mathrm{MoS}_{2}}{ }^{35}$ are found at about $2.1 \mathrm{eV}{ }^{2}$. Above the A excitons, corresponding spin-orbit split B excitations reside (only partially shown). Compared to the band gap of the $\mathrm{MoS}_{2}$ and $\mathrm{WS}_{2}$ layers this leads to exciton binding energies of about $0.5 \mathrm{eV}$ for $\mathrm{WS}_{2}$ and $0.6 \mathrm{eV}$ for $\mathrm{MoS}_{2}$, respectively. About $0.2 \mathrm{eV}$ below, another weak but optically active excitation is observed. To reveal its character, we evaluate the $z$-dependent exciton wave function

$$
\Phi^{S}\left(z_{h}, z_{e}\right)=\int d x \int d y \sum_{\mathbf{v c}} B_{\mathbf{v c}}^{S} \phi_{\mathbf{v}}^{*}\left(\mathbf{r}_{h}\right) \phi_{\mathbf{c}}\left(\mathbf{r}_{e}\right),
$$

\footnotetext{
${ }^{2}$ Note the we find the $\mathrm{MoS}_{2}$ exciton at slightly higher absorption energies in $G d W$ compared to experiment. 31
} 

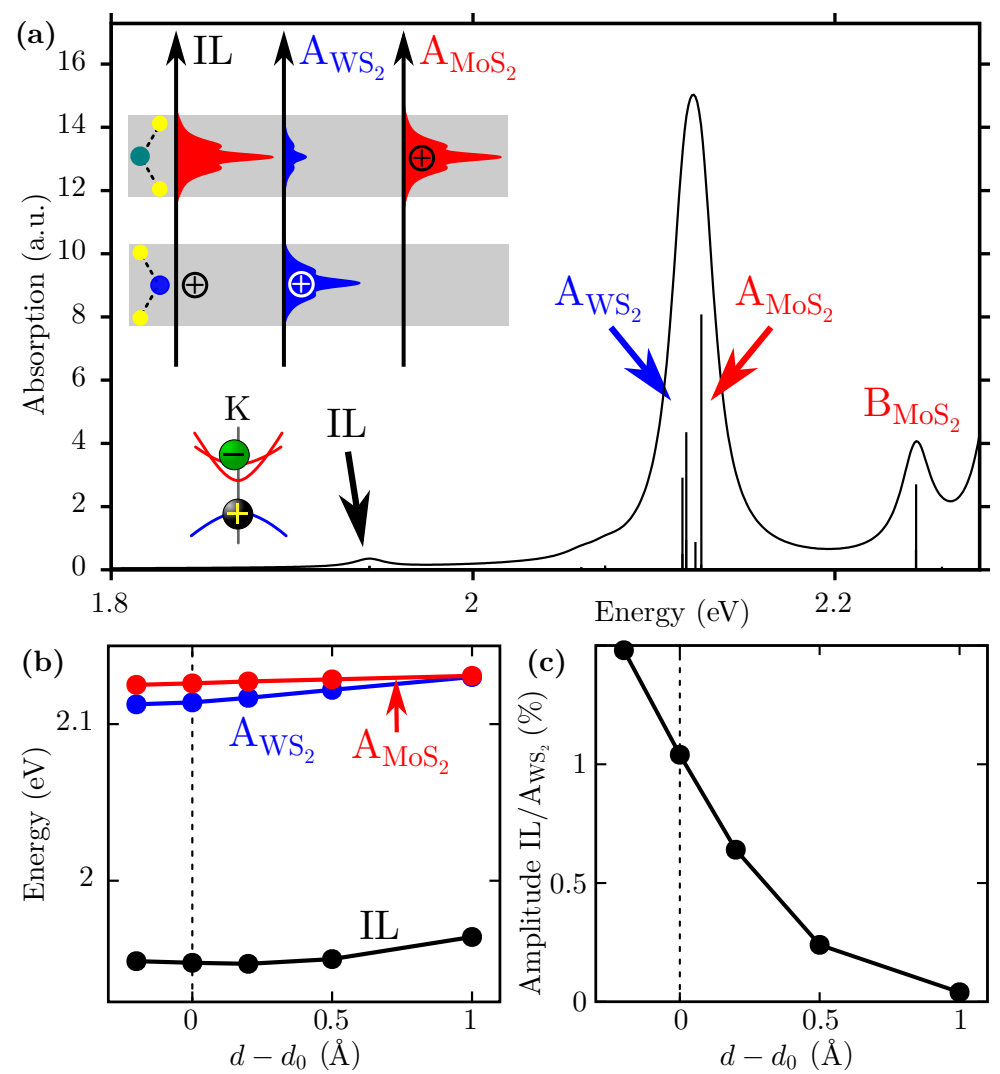

Figure 2: (a) Optical absorption spectrum of $\mathrm{MoS}_{2}-\mathrm{WS}_{2}$ heterostructure with interlayer (IL) and intralayer excitons $\left(\mathrm{A}_{\mathrm{WS}_{2}}, \mathrm{~A}_{\mathrm{MoS}_{2}}\right.$, and $\left.\mathrm{B}_{\mathrm{MoS}_{2}}\right)$. The inset show the electron probability perpendicular the heterostructure (see main text) and a sketch of the band structure with the strongest contributions indicated as electron and hole (band colors as in Fig. 1). (b) Energy dependence of the lowest three optical active excitations for different distances $d-d_{0}$. (c) Distance dependent ratio of the amplitude of IL and $\mathrm{A}_{\mathrm{WS}_{2}}$ exciton.

where $S$ labels the exciton. The coefficients $B_{\mathbf{v c}}^{S}$ have been obtained by the diagonalization of the Bethe-Salpeter equation (see supporting information). The energetically lowest state has strong contributions from both layers (see inset in Fig. 2(a)), i.e. the hole resides on the $\mathrm{WS}_{2}$ layer while the electron is mostly located on the $\mathrm{MoS}_{2}$ layer. Thus the exciton can be identified as an interlayer (IL) state. Compared to the direct gap at $\mathrm{K}$ we find an exciton binding energy of $0.35 \mathrm{eV}$. This is slightly lower than the intralayer excitons which results from the reduced electron-hole interaction due to its increased spatial distance. Furthermore this value is in good agreement with the value for the interlayer exciton in $\mathrm{MoS}_{2} / \mathrm{WSe}_{2}$ obtained for from simpler models $(0.3 \mathrm{eV}) . \stackrel{[26}{2}$ We note that the energetic ordering of the 

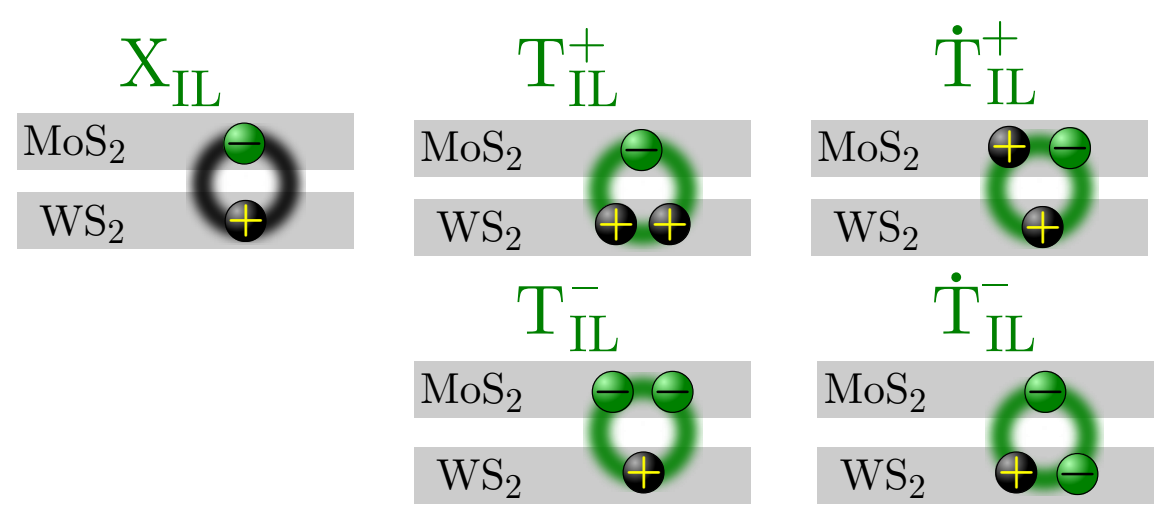

Figure 3: Possible spatial extent of interlayer trion states in a heterobilayer in comparison to the interlayer exciton $\mathrm{X}_{\mathrm{IL}}$. Four transitions with distinctly different character are possible, which are labeled $\mathrm{T}_{\mathrm{IL}}^{+}$and $\dot{\mathrm{T}}_{\mathrm{IL}}^{+}$for positive trions as well as $\mathrm{T}_{\mathrm{IL}}^{-}$and $\dot{\mathrm{T}}_{\mathrm{IL}}^{-}$for the negative case (see main text).

Table 1: Degeneracy of the possible exciton and trion states (compare Fig. 3). The fine splittings of different spin and valley configurations ${ }^{31}$ (remains 2 -fold degenerated) is ignored.

\begin{tabular}{c|cc|cc|cc} 
State & $\mathrm{A}$ & $\mathrm{X}_{\mathrm{IL}}$ & $\mathrm{T}_{\mathrm{IL}}^{+}$ & $\dot{\mathrm{T}}_{\mathrm{IL}}^{+}$ & $\mathrm{T}_{\mathrm{IL}}^{-}$ & $\dot{\mathrm{T}}_{\mathrm{IL}}^{-}$ \\
\hline Degeneracy & 2 & 2 & 2 & 4 & 6 & 8
\end{tabular}

intralayer peaks in the bilayer is reversed by about $10 \mathrm{meV}$ in comparison to our monolayer calculations which agree with experimental measurements. ${ }^{36}$ However, for the discussion of IL excitations the exact ordering of the bands is not important.

In Fig. 2(b) the energetic position for different interlayer distance $d-d_{0}$ of the two layers is shown. Overall the energetic positions of the excitons are quite insensitive to this distance 3. While the intralayer excitations of $\mathrm{MoS}_{2}$ and $\mathrm{WS}_{2}$ have a similar optical intensity, the IL state is drastically reduced in absorption. In Fig. 2(c) the dependence of the intensity on $d-d_{0}$ is shown. Relative to the intensity of the A excitons, the IL state is decreased by a factor of 25 when enlarging the distance by $1 \AA$. This can be understood from the smaller wave-function overlap of interlayer excitons compared to intralayer states.

Until now only neutral excitons have been discussed. When the system becomes doped, e.g. due to a substrate or gating, $\frac{16}{16}$ charged trions may form and can be visible in the optical

\footnotetext{
${ }^{3}$ In contrast, while the energetic position of the intralayer transitions in $\mathrm{MoS}_{2}$ almost constant, a minimal increase of the exciton energy is observed for $\mathrm{A}_{\mathrm{WS}_{2}}$. This shift can be explained due to the small contribution on the $\mathrm{MoS}_{2}$ layer for $\mathrm{AWS}_{2}$ (Fig. 2(a)) As the interlayer exciton is build from states on both layers, it shows a slightly larger response and for an enlarged distance of $1 \AA$ it shifts by 20 meV upwards in energy.
} 
spectrum. For the IL exciton the spatial structure is determined by the band alignment (Fig. 1): the hole resides on $\mathrm{WS}_{2}$ and the electron on $\mathrm{MoS}_{2}$ (see Fig. 3). In contrast to this, the positions of additional holes or electrons are not immediately clear. If two identical charges are localized on the same layer, (labeled $\mathrm{T}_{\mathrm{IL}}^{+/-}$) the repulsion between these particles might be large, while the opposite (labeled $\dot{\mathrm{T}}_{\mathrm{IL}}^{+/-}$) might be seen as neutral from the other layer. In Tab. 1 we show the degeneracy of the bright excitations. While the A exciton in $\mathrm{MoS}_{2} / \mathrm{WS}_{2}$ and the IL state are doubly degenerate (at $\mathrm{K}$ and $-\mathrm{K}$ valley), trion states may have a higher degeneracy due to the larger number of possible combinations for distributing three compared to two particles. Neglecting the tiny spin-splitting of the conduction band the degeneracies are $2 / 4$ for $\mathrm{T}_{\mathrm{IL}}^{+} / \dot{\mathrm{T}}_{\mathrm{IL}}^{+}$and $6 / 8$ for their negative counterparts, respectively.

To resolve the question which of the trions shown in Fig. 3 is the ground state, we employ the three-particle Hamiltonian 24

$$
\hat{H}^{(\text {hhe/eeh })}=\hat{H}_{\mathrm{BS}}+\hat{H}_{h h / e e}+\hat{H}_{e h, 1}+\hat{H}_{e h, 2}
$$

which consists of the single-particle band structure energies and the three-particle interactions (see Supplement). The eigenstates of this Hamiltonian represent the trion excitations. Their energies $E^{T}$ relative to the initial single-particle state $\epsilon_{v}$ are related to the photoexcitation energy via $\hbar \omega_{T \leftrightarrow v}=E^{T}-\epsilon_{v}$. In the optical spectrum bound states can be found below the excitons $\left(\hbar \omega_{X \leftrightarrow 0}\right)$ with an additional trion binding energy $E_{\mathrm{b}}^{T}=\hbar \omega_{X \leftrightarrow 0}-\hbar \omega_{T \leftrightarrow v}$. We observe positive and negative trions in the absorption spectra (depicted in green in Figs. $4(a, b)$ for the energetically lowest states). In addition to the neutral interlayer excitons discussed above we find further trion states $\mathrm{T}_{\mathrm{IL}}^{+}$and $\mathrm{T}_{\mathrm{IL},(1 / 2 / 3)}^{-}$, as well as $\dot{\mathrm{T}}_{\mathrm{IL},(1 / 2)}^{+}$with a small but non-vanishing amplitudes. The trion binding energy, i.e. the energetic difference between the IL exciton and trion in Figs. $4(\mathrm{a}, \mathrm{b})$, amounts to about $E_{\mathrm{b}}^{\mathrm{T}_{\mathrm{IL}}^{+}}=18 \mathrm{meV}$ and $E_{\mathrm{b}}^{\mathrm{T}_{\mathrm{IL}, 1}^{-}}=28 \mathrm{meV}$, respectively. The trions $\dot{\mathrm{T}}_{\mathrm{IL},(1 / 2)}^{+}$are found nearly at the same energy as the neutral IL exciton. We note that the number of observed trion states corresponding to 
the peaks is different for the $n$ - and p-doped materials (see Tab. 1).

The spatial structure of the trions can be deduced from their names (see Fig. 3) as well as from the color of the bands on the inset of Fig. 4 where we use blue and red for the bands of $\mathrm{MoS}_{2}$ and $\mathrm{WS}_{2}$, respectively. Furthermore, in Fig. 4(c) the real-space wave function for positive trions is shown. We have fixed the electron on the $\mathrm{MoS}_{2}$ layer and the hole probability is pictured after averaging over the coordinates of one of the two holes and integrating the in-plane directions (compare to insets in Fig. 2(a)). Interestingly, in the bound states the two identical particles reside on the same layer, e.g. for the $\mathrm{T}_{\mathrm{IL}}^{+}$trion, the two holes both reside on the $\mathrm{WS}_{2}$ layer with momenta distributed around the $\mathrm{K}$ and $-\mathrm{K}$ valleys in the Brillouin zone. If the identical particles reside on different layers (the case of the $\dot{\mathrm{T}}_{\mathrm{IL},(1 / 2)}^{+}$trions) we find a resonant character, i.e. the interlayer exciton and the hole on the other layer are hardly bound (thus its naming). We note that resonant states are not fully converged with the employed k-point mesh. For the negative trions we expect two similar resonant trion states $\dot{\mathrm{T}}_{\mathrm{IL},(1 / 2)}^{-}$, but due to the larger number of states 4 we were not able to converge them. Analogous to the positive trions we expect a similar vanishing binding energy of $\dot{\mathrm{T}}^{-}$if a neutral exciton and the electron are on different layers.

The interlayer trions $\mathrm{T}_{\mathrm{IL}}^{+/-}$are bound three-particle states, i.e. their excitation energy is below those of $\mathrm{X}_{\mathrm{IL}}$. A decomposition of the different contributions to the trions (Eq. (2)) reveals that the additional hole nearly doubles the electron-hole attraction from $\left\langle H_{e h}\right\rangle=$ $170 \mathrm{meV}$ to $310 \mathrm{meV}$, which clearly indicates the bound character of both holes. On the other hand a repulsive hole-hole interaction of $120 \mathrm{meV}$ is found, which drives the holes on the same layer apart from each other. The difference between these terms (additional $140 \mathrm{meV}$ attraction and $120 \mathrm{meV}$ repulsion, i.e. $20 \mathrm{meV}$ ) determines the trion binding energy and thus the position where it is found in the spectrum.

In general the signature of a trion can occur several times in the optical spectra (Fig.4(d)). This is due to the fact that the excitation energy of a trion $E^{T}$ is found with reference to

\footnotetext{
${ }^{4}$ In our calculations we find distinctly more negative than positive trions. This results from further states with particles residing in the second $\mathrm{CB}$ and the $\mathrm{CB}$ minimum between $\mathrm{K}$ and $\Gamma$.
} 

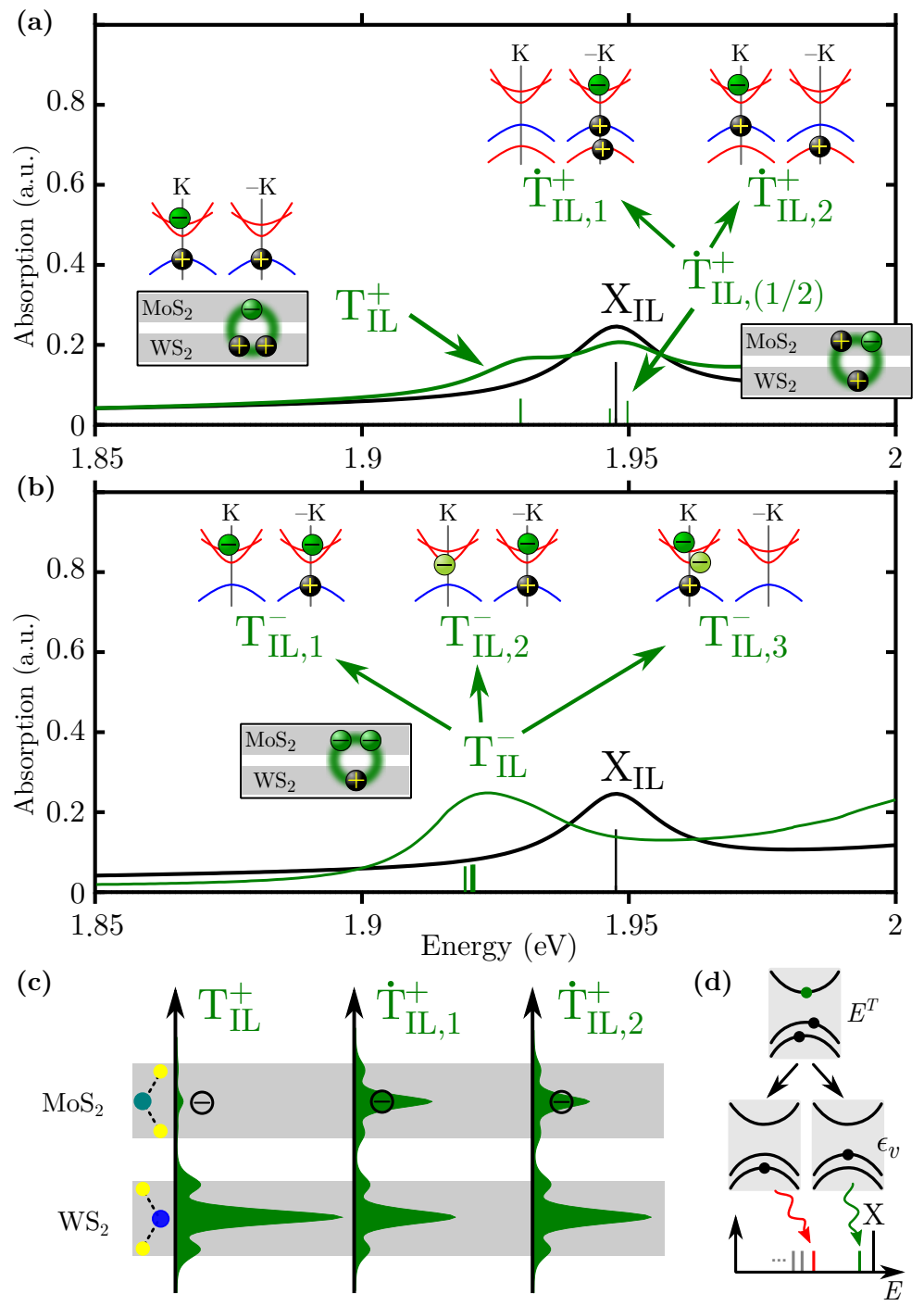

Figure 4: (a) Optical absorption spectrum for positively charged trions (green) and neutral excitons (black) for the energetically lowest states. A sketch of the band-structure contributions of electrons and holes to the bright intralayer trion $\mathrm{T}_{\mathrm{IL}}^{+}$and $\dot{\mathrm{T}}_{\mathrm{IL},(1 / 2)}$ is pictured as inset (see Fig. 2). (b) Optical absorption spectrum of negative trions (green) and neutral excitons (black). The three insets show the different contributions to the corresponding optical active trions. (c) Hole probability of $\mathrm{T}_{\mathrm{IL}}^{+}$and $\dot{\mathrm{T}}_{\mathrm{IL},(1 / 2)}$ after averaging over the coordinates of one of the two holes and integrating along both in-plane directions. The electron is fixed on the $\mathrm{MoS}_{2}$ layer. (d) Sketch of the recombination of a trion in two different single-particle states and resulting peaks in the spectrum (see main text). 
the involved single-particle state $\hbar \omega_{T \leftrightarrow v}=E^{T}-\epsilon_{v}$. For $\dot{\mathrm{T}}_{\mathrm{IL}}^{+}$the holes can be found on different layers and correspondingly the single-particle state $\epsilon_{v}$ can refer to $\mathrm{MoS}_{2}$ or $\mathrm{WS}_{2}$. Thus we find two peaks corresponding to a remaining hole on the $\mathrm{MoS}_{2}$ layer and a hole on the $\mathrm{WS}_{2}$ layer. While we find the first peak slightly above the $\mathrm{MoS}_{2}$ exciton (not shown), the second one resides close to the $\mathrm{X}_{\mathrm{IL}}$ (the $\mathrm{VB}$ difference is $0.36 \mathrm{eV}$ while the difference of exciton energies is about $0.2 \mathrm{eV}$ ). These energy differences indicate that $\dot{\mathrm{T}}_{\mathrm{IL}}^{+}$would most likely dissociate into $\mathrm{X}_{\mathrm{IL}}$ and a free hole on the $\mathrm{MoS}_{2}$ layer or $\mathrm{A}_{\mathrm{MoS}_{2}}$ and a free hole on the $\mathrm{WS}_{2}$ layer.

In comparison to the monolayers of $\mathrm{MoS}_{2}$ and $\mathrm{WS}_{2}$ the binding energies of the pure intralayer trions, i.e. with all three particles located in the same layer, are slight reduced in the bilayer. For negative/positive trions in monolayers (in vacuum) we obtain $E_{\mathrm{b}, \mathrm{MoS}_{2}}^{\mathrm{tr}}=$ $46 / 41 \mathrm{meV}$ and $E_{\mathrm{b}, \mathrm{WS}_{2}}^{\mathrm{tr}}=61 / 33 \mathrm{meV} \cdot \frac{37}{37}$ The reduction in comparison to the bare monolayer reflects the reduced Coulomb interaction due to the dielectric screening of the other layer and the spatially separated particles. Their character (one positive and three negative) is similar to intralayer trions. In a similar hetero-bilayer $\left(\mathrm{MoS}_{2} / \mathrm{MoSe}_{2}\right.$ placed on a substrate) Mouri et al. ${ }^{22}$ reported an upper limit of $\sim 25 \mathrm{meV}$ for the trion binding energy, which is in good agreement with our result. We note that for binding energy and amplitudes of the IL trions $\mathrm{T}^{+/-}$we find the same trends with respect to the layer distance as observed for IL excitons (see Fig. 2(b,c)).

In addition to the excitations discussed so far, which are all optically active (at $\pm K$ ), we find an large number of states with vanishing optical intensity. This includes trions composed of non-zero momentum excitons with electrons at the minimum of $\mathrm{K}-\Gamma$ (n doped samples) or holes at $\Gamma$. We note that we concentrate on the lowest lying states in this study, therefore bright states at higher energies or dark states of triplet character or finite total momentum will not be discussed further here 5 .

\footnotetext{
${ }^{5}$ The transition energy of trions in the optical spectrum is given by $\Omega(|T, \mathbf{K}\rangle \leftrightarrow|c \mathbf{K}\rangle)=E^{(T, \mathbf{K})}-\epsilon_{c \mathbf{K}}$, where $T$ counts the trions, $\mathbf{K}$ is it momentum of the trion as well as the final state of the remaining electron $|c \mathbf{K}\rangle$. Here we have focused on trions with the lowest transition energies with a momentum $\mathbf{K}=\mathrm{K}$.
} 


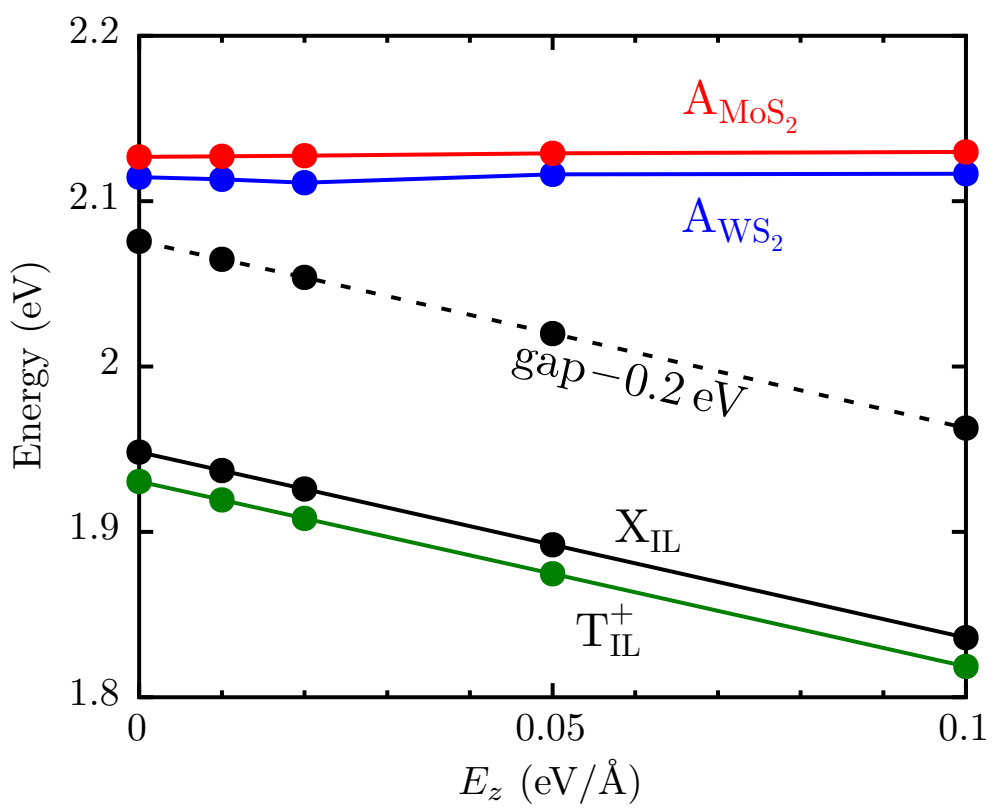

Figure 5: The $\mathrm{MoS}_{2}-\mathrm{WS}_{2}$ heterostructure in an electrical field $E_{z}$ perpendicular to the layers. The IL, $\mathrm{WS}_{2}$, and $\mathrm{MoS}_{2}$ exciton are shown in black, blue, and red, respectively. In addition the positive trion is depicted in green. The direct gap at $\mathrm{K}$ (black dotted line) is shifted down by $0.2 \mathrm{eV}$.

In this study we have considered, for computational simplicity, the IL trions in the specific heterobilayer $\mathrm{MoS}_{2}-\mathrm{WS}_{2}$ only. However, we expect the main conclusions to be valid for a range of other heterobilayers with Type II band alignment.

Depending on the experimental setup it might be difficult to determine if a peak corresponds to an inter- or intralayer state. The interaction with the second layer may also slightly modify the positions of the intralayer excitons and trions, e.g. the enhanced screening by the other layer may lead to a small red shift. ${ }^{31}$ While interlayer excitations in bulk materials have been identified by the sign of the corresponding $g$-factor, 10111 applying an electrical field perpendicular to the heterostructure is an alternative strategy. Applied electric fields and the resulting Stark effect have already been discussed extensively in the literature. Most often a quadratic Stark effect is observed, e.g. for quantum dots ${ }^{38139}$ or TMDCs (in an in-plane field) ${ }^{40141}$ On the other hand, in coupled quantum wells ${ }^{421}$ the linear Stark effect dominated and in single quantum wells the quantum-confined Stark effect $\underline{43}$ has been found. In TMDC heterostructures with a perpendicular electric field a linear Stark effect is expected similar 
to coupled quantum well systems.

In Fig. 5 the resulting shift of the exciton energies for different electric field strengths is shown. While the intralayer excitons $\mathrm{AWS}_{2}$ and $\mathrm{A}_{\mathrm{MoS}_{2}}$ are mostly constant (due to the smaller out of plane polarizability, the quadratic Stark effect is much smaller compared to in-plane fields), the IL state shifts down with increasing fields. This behavior can be easily understood by spatial structure of the excitons. The A excitons have almost all contributions on one layer, and consequently the energy of the hole and electron forming the exciton will shift in the same way by the electric field. In contrast the IL states have electrons and holes distributed on different layers. Hence their shift immediately follow those of the direct band gap (dotted line in Fig. 5) between the $\mathrm{VB}$ of $\mathrm{WS}_{2}$ and the $\mathrm{CB}$ of $\mathrm{MoS}_{2}$ at $\pm \mathrm{K}$. The fact that the exciton energy follows exactly the direct band gap as function of the field strength, indicates that the exciton binding energy remains unchanged. By inverting the electric field the direct gap and the IL states can be moved to higher energies as well. Note that these trends are only valid for states localized around $\pm \mathrm{K}$. If contributions stem from states around $\Gamma$ or $\Gamma-\mathrm{K}$ this will result in a different slope due to the stronger interlayer hybridization.

In summary, we have presented an ab-initio study of the low energy optical properties of a $\mathrm{MoS}_{2}-\mathrm{WS}_{2}$ heterostructure which are governed by the neutral excitons and charged trions. In addition to previously studied IL excitons we find IL trions with different spatial character. We identify the lowest binding energy of $18 / 28 \mathrm{meV}$ for positive/negative trions when both holes/electrons resides on the same layer. In contrast to this, a negligible binding energy is found for an electron/hole interacting with an intralayer exciton of the other layer.

\section{Acknowledgements}

T.D. acknowledges the financial support from the Villum foundation. The Center for Nanostructured Graphene (CNG) is sponsored by the Danish Research Foundation, Project DNRF103. 


\section{Supporting Information}

A detailed discussion of the applied theoretical methods to calculate excitons and trions; two figures showing the numerical convergence of excitons and trions with respect to the applied k-mesh (PDF)

\section{References}

(1) Mak, K. F.; He, K.; Shan, J.; Heinz, T. F. Nat. Nanotechnology 2012, 7, 494-498.

(2) Sallen, G.; Bouet, L.; Marie, X.; Wang, G.; Zhu, C. R.; Han, W. P.; Lu, Y.; Tan, P. H.; Amand, T.; Liu, B. L.; Urbaszek, B. Phys. Rev. B: Condens. Matter Mater. Phys. 2012, 86, 081301.

(3) Xiao, D.; Liu, G.-B.; Feng, W.; Xu, X.; Yao, W. Phys. Rev. Lett. 2012, 108, 196802.

(4) Yin, Z.; Li, H.; Li, H.; Jiang, L.; Shi, Y.; Sun, Y.; Lu, G.; Zhang, Q.; Chen, X.; Zhang, H. ACS Nano 2012, 6, 74-80.

(5) Srivastava, A.; Sidler, M.; Allain, A. V.; Lembke, D. S.; Kis, A.; Imamoğlu, A. Nat. Nanotechnology 2015, 10, 491-496.

(6) Koperski, M.; Nogajewski, K.; Arora, A.; Cherkez, V.; Mallet, P.; Veuillen, J.-Y.; Marcus, J.; Kossacki, P.; Potemski, M. Nat. Nanotechnology 2015, 10, 503-506.

(7) Eisenstein, J. P.; MacDonald, A. H. Nature 2004, 432, 691-694.

(8) Min, H.; Bistritzer, R.; Su, J.-J.; MacDonald, A. H. Phys. Rev. B: Condens. Matter Mater. Phys. 2008, 78, 121401.

(9) Li, Y.-M.; Li, J.; Shi, L.-K.; Zhang, D.; Yang, W.; Chang, K. Phys. Rev. Lett. 2015, $115,166804$.

(10) Tanaka, M.; Fukutani, H.; Kuwabara, G. J. Phys. Soc. Jpn. 1978, 45, 1899-1904. 
(11) Arora, A.; Drüppel, M.; Schmidt, R.; Deilmann, T.; Schneider, R.; Molas, M. R.; Marauhn, P.; Potemski, M.; Rohlfing, M.; Bratschitsch, R. Nat. Commun. 2017, 8, 639.

(12) Ceballos, F.; Bellus, M. Z.; Chiu, H.-Y.; Zhao, H. ACS Nano 2014, 8, 12717-12724.

(13) Gong, Y. et al. Nat. Mater. 2014, 13, 1135-1142.

(14) Rivera, P.; Seyler, K. L.; Yu, H.; Schaibley, J. R.; Yan, J.; Mandrus, D. G.; Yao, W.; Xu, X. Science 2016, 351, 688-691.

(15) Wilson, N. R.; Nguyen, P. V.; Seyler, K.; Rivera, P.; Marsden, A. J.; Laker, Z. P.; Constantinescu, G. C.; Kandyba, V.; Barinov, A.; Hine, N. D.; Xu, X.; Cobden, D. H. Sci. Adv. 2017, 3, e1601832.

(16) Mak, K. F.; He, K.; Lee, C.; Lee, G. H.; Hone, J.; Heinz, T. F.; Shan, J. Nat. Mater. 2013, 12, 207-211.

(17) Ross, J. S.; Wu, S.; Yu, H.; Ghimire, N. J.; Jones, A. M.; Aivazian, G.; Yan, J.; Mandrus, D. G.; Xiao, D.; Yao, W.; Xu, X. Nat. Commun. 2013, 4, 1474.

(18) Hao, K.; Xu, L.; Nagler, P.; Singh, A.; Tran, K.; Dass, C. K.; Schüller, C.; Korn, T.; Li, X.; Moody, G. Nano Lett. 2016, 6b02041.

(19) Plechinger, G.; Nagler, P.; Arora, A.; Schmidt, R.; Chernikov, A.; del Águila, A. G.; Christianen, P. C. M.; Bratschitsch, R.; Schüller, C.; Korn, T. Nat. Commun. 2016, 7, 12715 .

(20) Mouri, S.; Miyauchi, Y.; Matsuda, K. Nano Lett. 2013, 13, 5944-5948.

(21) Mouri, S.; Zhang, W.; Kozawa, D.; Miyauchi, Y.; Eda, G.; Matsuda, K. Nanoscale 2017,6674 . 
(22) Miller, B.; Steinhoff, A.; Pano, B.; Klein, J.; Jahnke, F.; Holleitner, A.; Wurstbauer, U. Nano Lett. 2017, 7b01304.

(23) Rohlfing, M.; Louie, S. G. Phys. Rev. B: Condens. Matter Mater. Phys. 2000, 62, 4927-4944.

(24) Deilmann, T.; Drüppel, M.; Rohlfing, M. Phys. Rev. Lett. 2016, 116, 196804.

(25) Nayak, P. K.; Horbatenko, Y.; Ahn, S.; Kim, G.; Lee, J.-U.; Ma, K. Y.; Jang, A.-R.; Lim, H.; Kim, D.; Ryu, S.; Cheong, H.; Park, N.; Shin, H. S. ACS Nano 2017, 11, 7b00640.

(26) Latini, S.; Winther, K. T.; Olsen, T.; Thygesen, K. S. Nano Lett. 2016, 17, 6b04275.

(27) Kośmider, K.; Fernández-Rossier, J. Phys. Rev. B: Condens. Matter Mater. Phys. 2013, 87,075451 .

(28) Yun, W. S.; Han, S. W.; Hong, S. C.; Kim, I. G.; Lee, J. D. Phys. Rev. B: Condens. Matter Mater. Phys. 2012, 85, 033305.

(29) Böker, T.; Severin, R.; Müller, A.; Janowitz, C.; Manzke, R.; Voß, D.; Krüger, P.; Mazur, A.; Pollmann, J. Phys. Rev. B: Condens. Matter Mater. Phys. 2001, 64, 235305.

(30) Rohlfing, M. Phys. Rev. B: Condens. Matter Mater. Phys. 2010, 82, 205127.

(31) Drüppel, M.; Deilmann, T.; Krüger, P.; Rohlfing, M. Nat. Commun. 2017, 8, 2117.

(32) Winther, K. T.; Thygesen, K. S. 2D Materials 2017, 4, 025059.

(33) Strinati, G. Phys. Rev. Lett. 1982, 49, 1519-1522.

(34) Onida, G.; Reining, L.; Rubio, A. Rev. Mod. Phys. 2002, 74, 601-659.

(35) Qiu, D. Y.; da Jornada, F. H.; Louie, S. G. Phys. Rev. Lett. 2013, 111, 216805. 
(36) Low, T.; Chaves, A.; Caldwell, J. D.; Kumar, A.; Fang, N. X.; Avouris, P.; Heinz, T. F.; Guinea, F.; Martin-Moreno, L.; Koppens, F. Nat. Mater. 2016, 16, 182-194.

(37) Deilmann, T.; Thygesen, K. S. Phys. Rev. B: Condens. Matter Mater. Phys. 2017, 96, 201113.

(38) Krenner, H. J.; Sabathil, M.; Clark, E. C.; Kress, A.; Schuh, D.; Bichler, M.; Abstreiter, G.; Finley, J. J. Phys. Rev. Lett. 2005, 94, 057402.

(39) Stinaff, E. A.; Scheibner, M.; Bracker, A. S.; Ponomarev, I. V.; Korenev, V. L.; Ware, M. E.; Doty, M. F.; Reinecke, T. L.; Gammon, D. Science 2006, 311, 636-639.

(40) Pedersen, T. G. Phys. Rev. B: Condens. Matter Mater. Phys. 2016, 94, 125424.

(41) Haastrup, S.; Latini, S.; Bolotin, K.; Thygesen, K. S. Phys. Rev. B: Condens. Matter Mater. Phys. 2016, 94, 041401.

(42) Le, H. Q.; Zayhowski, J. J.; Goodhue, W. D. Appl. Phys. Lett. 1987, 50, 1518-1520.

(43) Miller, D. A. B.; Chemla, D. S.; Damen, T. C.; Gossard, A. C.; Wiegmann, W.; Wood, T. H.; Burrus, C. A. Phys. Rev. Lett. 1984, 53, 2173-2176. 
Graphical TOC Entry

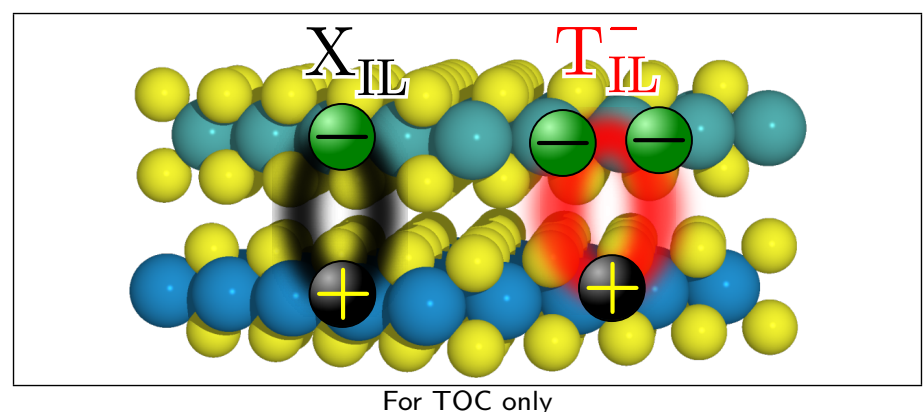

